Cserkaszky, Aron, Kara, Peter, Tamboli, Roopak R., Barsi, Attila, Martini, Maria G. and Balogh, Tibor (2018) "Light-field capture and display systems : limitations, challenges, and potentials", Novel Optical Systems Design and Optimization XXI, Proceedings of SPIE Volume 10746, ISSN 0277-786X (2018) https://

doi.org/10.1117/12.2320564. One print or electronic copy may be made for personal use only. Systematic reproduction and distribution, duplication of any material in this paper for a fee or for commercial purposes, or modification of the content of the paper are prohibited. 


\title{
Light-field capture and display systems: limitations, challenges and potentials
}

\author{
Aron Cserkaszky ${ }^{\mathrm{a}}$, Peter A. Kara ${ }^{\mathrm{b}}$, Roopak R. Tambolia ${ }^{\mathrm{a}}$, \\ Attila Barsi ${ }^{\mathrm{a}}$, Maria G. Martini ${ }^{\mathrm{b}}$, and Tibor Balogh ${ }^{\mathrm{a}}$ \\ ${ }^{a}$ Holografika, Budapest, Hungary \\ ${ }^{\mathrm{b}}$ WMN Research Group, Kingston University, Kingston upon Thames, UK
}

\begin{abstract}
The recent advances in light-field acquisition and display systems bring closer the day when they become commercially available and accessible to wide audiences for numerous use cases. Their usefulness and potential benefits have already been disseminated in the field and they started emerging in both industry and entertainment applications. The long-term goal of the scientific community and future manufacturers is to research and develop fully immersive, yet seamless and efficient systems that can achieve the ultimate visual experience. However, certain paths leading to such goals are blocked by technological and physical limitations, and also significant challenges that have to be coped with. Although some issues that rise regarding the development of capture and display systems may actually be nearly impossible to overcome, the potential for light-field applications is indeed immense, thus worth the vast scientific effort. In this paper, we systematically analyze and present the current and future relevant limitations and challenges regarding the research and development of light-field systems. As current limitations are primarily application-specific, both challenges and potentials are approached from the angle of end-user applications. The paper separately highlights the use case scenarios for industry and entertainment, and for everyday commercial usage. Currently existing light-field systems are assessed and introduced from a technical perspective and also with regards to usability, and potential future systems are described based on state-of-art technologies and research focuses. Aspects of practical usage, such as scalability and price, are thoroughly detailed for both light-field capture and visualization.
\end{abstract}

Keywords: light-field, glasses-free 3D visualization, technological challenges

\section{INTRODUCTION}

Light-field technology is slowly but steadily emerging, opening up a novel way of natural 3D visualization. Both light-field acquisition and display systems have already commercially surfaced, yet they still have a long way of technological evolution ahead of them. Possibly the most attractive property of light-field visualization is that absolutely no viewing gear is required to fully experience the capabilities of such systems, in comparison to other 3D technologies. The success of display systems evidently depend on capture systems as well, especially since certain applications may simultaneously rely on both (e.g., light-field telepresence). Both capture and display systems have great potentials - separately and together as well - yet wholly reaching these potentials may prove to be a difficult task, due to numerous limitations and challenges.

In this paper, we discuss the limitations, challenges and potentials regarding light-field capture and display systems. In the elaborated context, a limitation is something that cannot be properly tackled scientifically, due to physical, optical or other restrictions. The nature of fundamental limitations must be accepted and they must be kept in mind during research and also during application design, in order to avoid futile efforts and wasted

Further author information: (Send correspondence to Aron Cserkaszky)

Aron Cserkaszky: E-mail: a.cserkaszky@holografika.com

Peter A. Kara: E-mail: p.kara@kingston.ac.uk

Roopak R. Tamboli: E-mail: r.tamboli@holografika.com

Attila Barsi: E-mail: a.barsi@holografika.com

Maria G. Martini: E-mail: m.martini@kingston.ac.uk

Tibor Balogh: E-mail: t.balogh@holografika.com 
resources. Challenges on the other hand can be coped with; they can be addressed and they can be eliminated from the path leading to the long-term goals of the field. Yet they pose significant difficulties, some of which might be nearly impossible to handle. However, the necessary efforts are truly worth it, due to the potentials this technology holds. Potentials include the various applications in real-life scenarios and the many ways they can benefit humanity.

The reminder of the paper is structured as follows: the limitations, challenges and potentials of light-field technology are separately introduced in Section 2, 3 and 4, respectively. The paper is concluded in Section 5.

\section{LIMITATIONS}

On the level of terminology, light-field ${ }^{1}$ is a four-dimensional space where light rays are described on a convex surface emitted outwards in all directions. The color of the light rays is handled by having separate light-fields for the prime colors: red, green and blue. Light-fields are the practical form of the aim to describe the lights of a scene from every point, going to every directions, assuming a non-participating media — like clear air — to simplify lights of a scene by measuring light rays on a convex surface encompassing the scene. Light-fields have many parameterizations, such as the intersection of the rays with two defined planes, the intersection with a plane and two angles describing the direction of the rays, and the intersection of the rays with a sphere resulting in two angular coordinates in the spherical coordinate systems for both the entry and exit intersection.

An important property of light-fields is their baseline, which is the maximum distance between the change of perspective within the light-field. If the baseline extends to both vertical and horizontal directions, then the light-field is called full-parallax (FP), but as the vertical perspective of human observers changes less frequently than the horizontal one, light-field systems often have horizontal-parallax-only (HPO) light-fields. Light-field capture systems have an inherent baseline extending between the extremes of their captured images. For display systems, baseline is the distance between the extremes of the Field of View (FOV) in the typical observer display distance.

Admittedly the definition of the light-field already poses limitations. As the wavelength dimension is not described, it can only handle colors in the visible spectrum by exploiting the fact that the Human Visual System (HVS) has a very limited number of measured colors. Polarization and coherence of the light-rays is omitted for simplicity as well, therefore lasers and polarization effects can not be handled by it.

The convex surface restriction is necessary to avoid describing the same rays multiple times and to reduce the dimensionality to 4D. However, this means that views of the light-field can only be rendered outside of this convex surface, and the viewer can not enter the inside of the scene. This limitation of the light-field definition can be handled by choosing appropriate scenes and a minimal encompassing convex surface around it.

A physical limitation related to the fixed surface is that resolutions of real capture or display systems can not exceed the wavelength of visible light. This limitation does not effect current practical light-field systems, but might have an impact on future systems. Yet even before reaching this limit, we have to consider that all light-field displays have an inherently limited amount of rays they can emit. The distribution of these finite amount of rays in the $4 \mathrm{D}$ ray space are going to determine the FOV, the spatial, the angular and the depth resolution. The least intuitive of these parameters is the depth resolution, which is determined by density and spreading angle of the individual rays, as the virtual objects farther from the screen will be represented by more spread out rays, decreasing the perceived resolution at that depth level. This depth blurring effect manifests itself for all objects sufficiently far from the screen, for both foreground and background virtual objects. This limits the use cases of light-field displays for scenarios where the shown scene has a finite size, and is preferably within the volume of sufficient depth resolution of the display.

Capturing real light-field with cameras has limitations as placing large amount of cameras surrounding a scene is bound to create instances of self-capture, when some cameras of the system record images containing other parts of the systems. In practice, this means that the FOV of light-field capture systems is less than $180^{\circ}$. Analogously to this, light-field display systems have inherent limitations for valid observer positions. Depending on the FOV and resolution parameters of the display, there is a volume where virtual objects of the scene are placed, and observers have to be outside of this virtual volume. An additional difficulty is to arrange the observer area for front-projection systems. These are systems that illuminate a reflective screen from the same direction 
the observers see the screen - typically from above the observers - therefore an additional invalid observing area is created where the observer would cast a shadow on the screen. As an example, during the usage of the HoloVizio C80 front-projection cinema system, ${ }^{2}$ the position of the optical engine array defines the edge of recommended observing area; viewing the screen from closer distances may result in the occlusion of light rays, depending on observer height.

\section{CHALLENGES}

The most obvious challenge of light-field systems is to efficiently manage the immense data requirements of the $4 \mathrm{D}$ ray space. The two additional dimensions compared to traditional image technologies result in a 2 to 4 orders of magnitude increase in visual information. As a consequence, the scalability of light-field systems are challenged by capturing, rendering and displaying this vast amount of rays. Besides the price, weight and physical size of the many cameras for capture systems, and dense array of ray generators for display systems, there are many computing components required with their own resource footprints regarding power requirements, heat generation, bandwidth usage and storage space.

Current commercially available digital light-field display systems use a dense array of projectors as ray generators. ${ }^{3}$ These require graphical processing units (GPUs) to render the light-field and output the image to the projectors, and these GPUs are housed in nodes of a computer cluster, connected by network hardware with the required throughput capability. Regarding scalability, a GPU can typically render image for 2-4 projectors, and a computer node can commonly house 2,3 or at most 4 GPUs. The typical wide-baseline HPO light-field display systems have 60-100 projectors, 10-30 GPUs and 4-10 computer nodes, depending on their resolution parameters, FOV and baseline.

Capture systems are either one-camera-based with a microlense array providing a short-baseline FP lightfield, ${ }^{4}$ or use an array of cameras for wide-baseline light-field. The one-camera capture systems are relatively portable as a result of their limited baseline. Camera-array-based capture systems suffer similar limitations mentioned for display systems, as the camera rig can consist of 50-200 individual cameras and can be arranged linearly, or on a 2D grid. Often these individual cameras are providing raw frames through their gigabit ethernet or USB 3.0 connector to computer nodes that can compress, save and transmit the video feed. Ethernet is better for scaling, as cameras with those connectors usually transmit their unique identifiers, which helps in properly ordering the cameras.

Complex light-field systems that transmit the light-field of a capture system to be shown on a light-field display system are especially demanding on both the computing resources and bandwidth requirements, since for use cases such as 3D teleconferencing, both the system latency has to be low and the required bandwidth has to be reasonable. All these engineering challenges are hard to meet within a reasonable resource footprint and budget.

Compression of the entire light-field is a hot research topic and would help reducing the bandwidth and storage requirements of light-field systems. Existing $2 \mathrm{D}$ video compression methods are very efficient and can be used to compress camera feed streams, the videos of individual microlenses, and also the rendered output of the optical engines. However, this does not exploit the fact that the neighboring views of the light-field are highly correlated, therefore the compression ration is suboptimal. Initial attempts at compression the entire light-field have been using a static image of a microlense-array-based light-field camera, and treated the individual microlense images as frames of a video, and compressed it with existing $2 \mathrm{D}$ compression methods tailored for this new purpose. ${ }^{5}$ These methods gain in compression ratio but can only handle a static light-field image, as they use the temporal dimension of the compressed video for the spatial location of the subimages. This can be tackled by the use of 3D extensions of video codecs, that aim to compress multiple correlated video streams into a single highly compressed multi-view video. ${ }^{6}$ Yet it has been found that for practical use-cases, these initial implementation of multi-view encoding suffer issues regarding flexibility with differing resolutions and partial decoding. ${ }^{7}$

Another great challenge is the reconstruction of sparsely sampled light-fields into dense light-fields. Due to the already mentioned practical limitations, we can only have limited amount of samples from the entire light-field and there are use-cases where we need denser samples than practically achievable. A prime use-case example for this is when a captured light-field is shown on a light-field display, since the capture systems measure the 
light-field at specific and limited samples, and the display systems also has specific and limited rays that it can emit. Even if these two separate light-fields match in their main characteristics - i.e., FOV, number of samples, angular and spatial resolutions - the samples will not directly match in the 4D ray space. Designing such matching complex system is possible, however, manufacturing it with sufficient precision would be impractically hard. So in practice, complex systems use some form of interpolation in the $4 \mathrm{D}$ ray space to assign color to the display light-field rays, from the available neighboring samples of the capture light-field. ${ }^{8}$ An improvement on this is method is to render views from virtual cameras between views of real cameras and use this light-field to perform a higher quality interpolation. ${ }^{9}$ However, full dense reconstruction of the light-field is the ideal solution, as that ensures that there is no need for interpolation and there are no interpolation errors. Full reconstruction methods are only feasible for offline use-cases due to their high computational cost, but they have superior image quality. ${ }^{10}$ Another promising research area of dense light-fields is the free-viewpoint TV, which aims to render arbitrary views of captured events for the pleasure and immersion of viewers. ${ }^{11}$

Interchange formats of the light-fields between capture and display systems are also a topic of research as an efficient format can ease the computational cost of conversion between light-fields and should also allow broadcasting of this new media between any capture system to any or many different display systems in realtime, thereby allowing light-field teleconferencing systems to appear on the market. The goal of an interchange format is to create a generic mezzanine representation of the light-field ${ }^{12}$ that is not specific to any light-field system, but can describe their light-field well in most cases.

A practical engineering challenge of light-field systems is to create the capturing or displaying apparatus of the vast amount of light-field rays in a reasonable physical size and with an affordable budget. The physical size of the systems can not be shrunk to the same level as existing 2D based technologies without loosing the distinct advantages of light-field, but the smaller their size, the more use-cases they can allow. The current high price is also a barrier to market entry for many applications.

Another important engineering task regarding light-field systems is their calibration. Both capture and display systems have to have their rays precisely described, placing each ray into the 4D coordinates of the light-field. Then a display system is able to render the scene into the light-field and assign the appropriate color to each emitted ray. Likewise, a capture system can transform the measured color values into the $4 \mathrm{D}$ light-field ray space. The standard practice for calibrating a display system is to show special calibration patterns on the screen, which is observed by one or more cameras and the system can determine the alignment of each emitted ray. The capture system calibration is a similar process where a known calibration pattern is shown to the cameras and then each rays alignment is computed. Another important issue for both systems is color calibration is that all rays of the display have to have the same color response for the same rendered color, and cameras of capture systems have to measure the same color for the same perceived color. The temporal stability and sensitivity of the calibration mainly depends on the quality of its electronic components and the systems supporting structure to maintain its calibrated state despite physical actions, and other changes of the environment, such as temperature or humidity. This is especially challenging for moving and outdoor systems.

Existing algorithms and technologies for traditional 2D media are seldom useful light-fields. Regarding graphics and rendering, even simple things like tone mapping, ambient occlusion and screen space reflections that we already take for granted for 2D rendering - are completely different and not much research effort is going towards these areas. Broadcasting and transmission is also an area where existing solutions are lacking, and the challenge is to recreate these capabilities with intense research for light-fields. Finally, we have to select use-cases for light-field systems that no only take into account the inherent limitations of the technology, but also consider the current state of solutions for the aforementioned challenges.

\section{POTENTIALS}

In the previous two sections, we have reviewed all that makes the technological progress of light-field acquisition and visualization difficult and challenging. In this section, we elaborate what overcoming those challenges can achieve; we discuss the rewards of scientific efforts. The primary goal is to widely enable resource-efficient light-field systems for both industry and regular users. 

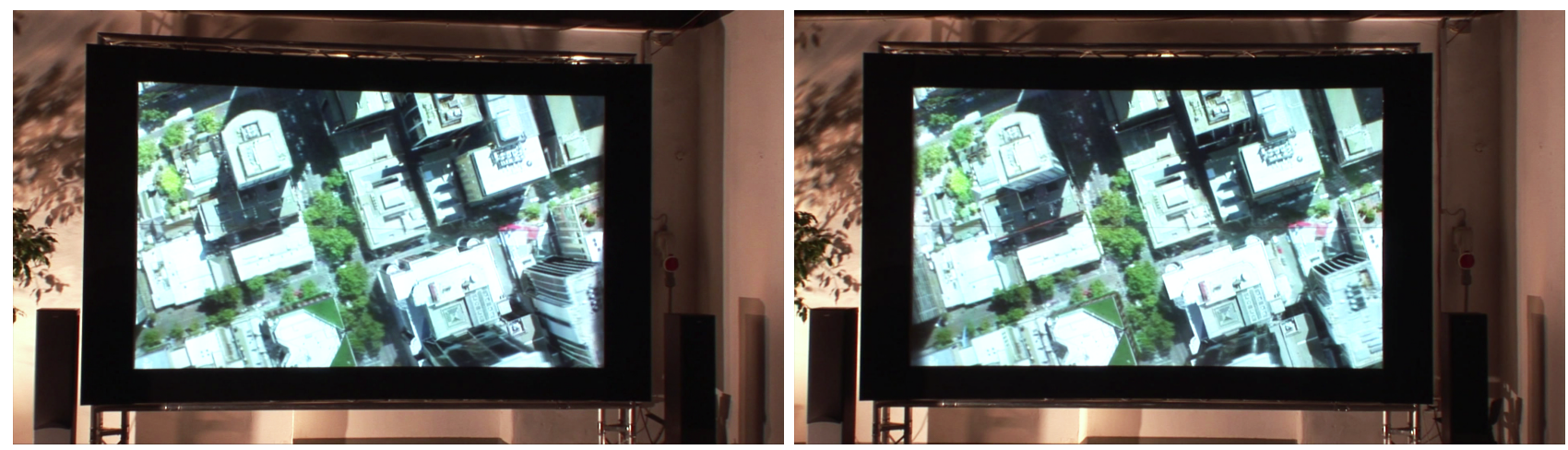

Figure 1. Visualization of myVR on the C80 cinema system, showing an aerial view of London.

An area where 3D imaging is already contributing a lot is the medical sector. Current 3D medical capture and visualization techniques definitely enhance efficiency and accuracy, compared to 2D diagnostics and surgical planning. ${ }^{13}$ Evidently, the extra dimension in visual information assists the work of medical experts, ${ }^{14}$ however, it needs to be noted that most of 3D medical imaging is viewed on 2D screens. This means that visualization is always limited a single view at a time, even though multiple optical operations are available on the medical content, such as rotation and zooming. There are also solutions which involve viewing devices, such as special 3D glasses, introducing the less natural, cumbersome nature of such devices. Having glasses-free 3D visualization at the disposal of doctors not only reduces the cognitive load that comes with the composition of numerous perceived 2D views into a 3D model, but also reduces the amount of interactions required to create an accurate, reliable medical diagnosis. The greatest relevance of this potential is during time-critical medical actions, such as preparation for an urgent operation.

A different professional area where the state-of-the-art situation is rather similar is 3D design. Design can target anything from buildings and vehicles of immense proportions to devices and components of miniature, microscopic sizes (e.g., reflecting on the medical use cases, a surgical probe that enters the human body). Similarly, the 3D models of a design are typically viewed on 2D screens or are observed with the help of viewing gears. Although the task of design can be commonly less time-critical than medical scenarios, still, increasing task efficiency ${ }^{15}$ can significantly benefit the process.

The enhanced levels of visual accuracy can contribute a lot to the exploration of the surface of the planet for resources. The most common resources in this context are gas and oil. ${ }^{16}$ Reliable mapping leads to better planning, resulting in high-efficiency resource extraction.

There are also important potentials for traffic control, both ground, sea and air. Particularly in the case of air traffic control, vehicle altitudes can be proportionally visualized, increasing the overall level of safety. As for navigation on a more personal level, light-field-based head-up displays in civilian vehicles can visualize navigational contents, i.e., 3D aerial views. An example of such content is shown on Figure $1^{*}$. A very similar application of light-field visualization in a different sector is the navigation of military assets. High-level control also applies, but rather for unit positioning than traffic control.

All previous use cases share the benefits of having multiple simultaneous viewers. The application where the support for simultaneous users is needed the most is public exhibition. During such, many attendees of the exhibition may wish to view the given passive or interactive visualization, that can be a model or even an entire scene. To accommodate a larger number of observers, a greater valid FOV is required, and depending on the content and the viewing distance, the resolution values of the system (spatial, angular and depth) must be higher as well. Generally speaking, such systems have notable potentials in education, from elementary school to university.

From all areas of potentials, probably the most relevant to regular users are communication and entertainment. In a way, these are the areas where unit cost matters the most, since the presence on the consumer market can only be ensured if and only if devices and services are affordable to a wide array of customers.

\footnotetext{
*myVR Software www.myvr-software.com/
} 

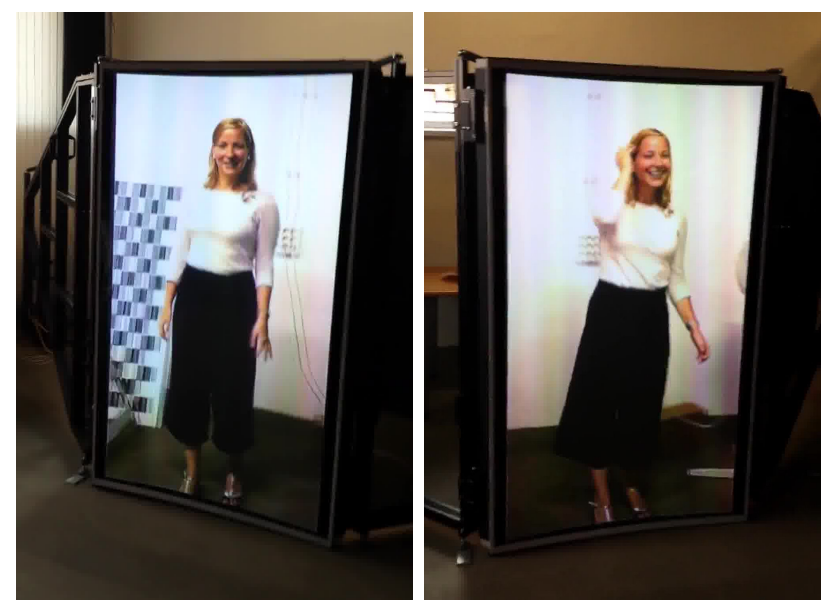

Figure 2. The telepresence system of Holografika in operation.

Since the beginning of personal telecommunication services, technology was thriving towards the so-called "sense of presence". It means that an end-user in a given location can experience the presence of another user in a remote location in audiovisual ways, tackling the physical distances that divides us. In the past decades, high-quality videoconferencing emerged in everyday life; today, people can hear and see each other in real time, with great sound and visual quality. Yet this is not the final frontier of the sense of presence. The absolute ultimate goal would be to have a representation of another human being that could not be discriminated from reality.

The next logical step on the path towards this goal is having a telepresence system that offers a life-size visualization of the user in glasses-free 3D. With light-field technology, such system is feasible. Figure 2 shows the display of the telepresence system of Holografika during testing, from different angles. The research leading to the construction and deployment of this system is currently being published.

At the time of this paper, the most rapidly growing part of entertainment is gaming. This paper does not aim to review the countless statistics that analyze the average number of hours weekly spent on gaming or the vast resources allocated to professional gaming events; the dominance of gaming within the entertainment industry is becoming undeniable. Instead, we discuss what potentials light-field visualization has for gaming.

First of all, as light-field systems are capable of having angularly-selective content visualization, it is possible to completely eliminate the issues that are present today for split-screen gaming. It was one of the earliest forms of multiplayer gaming, and it is still present today, although lost popularity parallel with the spread of high-speed Internet connection. The greatest value of this gaming method is the the sense of actual presence; the proximity of the other player. A major issue rises from the very definition of split-screen gaming: only a portion of the screen size is allocated to a given player, either with vertical or horizontal separation. As an example, in case of four simultaneous players, a mere quarter of the screen is used by a player. Yet a player has visual access to the entire screen, creating the next serious issue of competitive gameplay: screen-cheating or screen-peeking, which means that a player gains advantage in the game from the information that can be extracted from the portion of the screen allocated to the other player. With the angular allocation of gaming views to different players, both issues are dealt with; within a given interval of the valid FOV, the player only sees the correctly allocated information, shown on the entire screen of the light-field display.

Similarly to virtual reality gaming, it is expected that this visualization technology will have its own specific gaming genres. Although the potential is indeed huge, there are certain game types that will be rather challenging to accommodate on light-field systems. Open-world adventure games and outdoor first-person games (i.e., shooters) cannot be properly implemented without constrains, as the view of the player can point towards distances that are not possible to render in light-field. However, this can be handled in several ways, e.g., creating a game with strictly indoor settings, thus limiting the distance. Such issue is not present for turn-based and real-time strategy games, as the player looks down on the battlefield from a given position above all, with a constant distance. Figure 3 shows the gameplay of a real-time strategy game on a light-field display. 


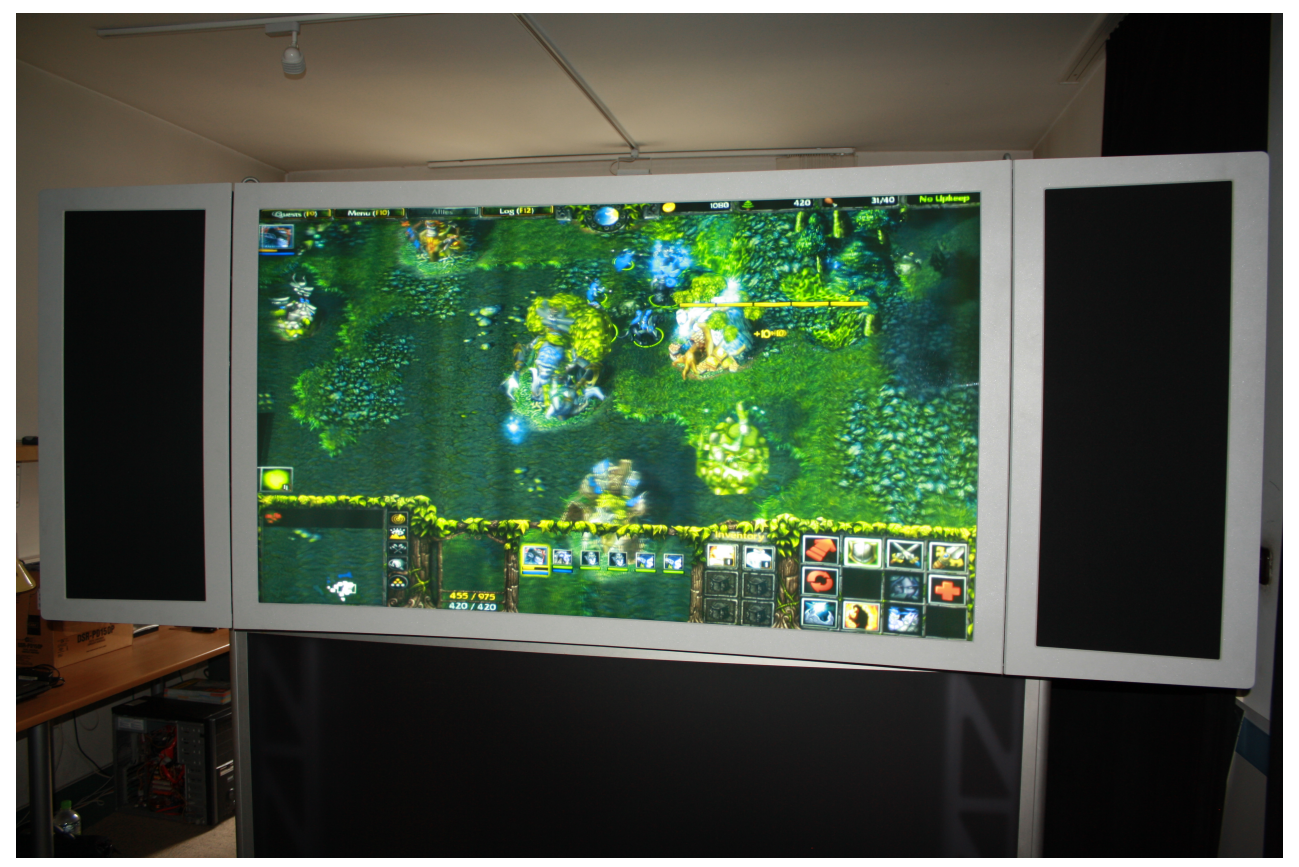

Figure 3. Warcraft III (C)Blizzard Entertainment) gameplay on the HoloVizio HV640.

The other major source of modern digital entertainment is video watching, which includes local playback and network streaming as well. In the previous sections, we have already discussed the difficulties of recording dynamic scenes, but let us just imagine a studio trying to record an outdoor car chase with a massive camera array. Of course rendered contents - such as animated movies - do not have to deal with such issues, but a home entertainment technology will not prevail on the market if it is limited to a single type of content. Recording movies will not solely rely on those who provide the technology, but also on the creativity of directors and content producers.

Speaking of which, creativity is highly supported by the novel frontiers of light-field cinematography. For instance, in a light-field cinema, ${ }^{17}$ the fact that different viewers see the content from different angles can be exploited for content-related and artistic purposes (e.g., some scene details may be visible from one angle, but occluded from another). Contents filmed in such ways may not only create a variation in the visual experience of the viewers, but also in other components of the global experience (e.g., emotional experience, if a tear running down the face of an actor/actress is only visible from a given segment of the valid FOV).

As for an actual light-field cinema, large-scale light-field displays already exist at the time of this paper, and upscaling the current state-of-the-art technology to the equivalent of a conventional 2D cinema today does not pose a theoretical challenge. The reflective screens of front-projection systems are straightforward to increase, as they do not have an internal structure nor active components. However, in practice, its physical manufacturing is a major challenge, and the deployment of such systems is further complicated by the issues of heat dissipation, power consumption and cost.

As it has been stated among the challenges, transmitting light-field video data over real networks with reasonable delay is a difficult task. Yet the concept of dynamic adaptive streaming not only applies to light-field as well, but it can be further extended with the parameter of content angular resolution, ${ }^{18}$ potentially lowering the chance of playback interruptions via network load reduction.

Lastly, it needs to be mentioned that the potentials we see today are those that we can imagine while relying on the current state-of-the-art and common use cases. The emergence of light-field capture and display applications in the industry and everyday life might evoke certain forms of utilizations that do not seem relevant today, but might become essential in the future. 


\section{CONCLUSION}

In this paper, we presented an analysis of the limitations, challenges and potentials of light-field capture and display systems. One conclusion of the presented aspects is that there is an evident need for such glasses-free 3D visualization technology, but it can only be delivered to the average user once the major challenges have been tackled with. Regarding the limitations, the majority of them shall always apply, but they shall not prevent the long-term development and deployment of efficient utilizations and applications. One may argue that despite all the scientific effort, light-field technology will never breach a point where it can surface and spread. As the opposite statement cannot be surely guaranteed, we can only say the limitations are given, the potentials are partially explored already, and in between the challenges must be addressed, so that one day natural glasses-free $3 \mathrm{D}$ can benefit humanity.

\section{ACKNOWLEDGMENTS}

The work in this paper was funded from the European Union's Horizon 2020 research and innovation program under the Marie Sklodowska-Curie grant agreements No 676401, European Training Network on Full Parallax Imaging and No 643072, Network QoE-Net.

\section{REFERENCES}

[1] Lippmann, G., "Epreuves reversibles. photographies integrals," Comptes-Rendus Academie des Sciences 146, 446-451 (1908).

[2] Balogh, T., Nagy, Z., Kovács, P. T., and Adhikarla, V. K., "Natural 3D content on glasses-free light-field 3D cinema," in [ISET/SPIE Electronic Imaging], International Society for Optics and Photonics (2013).

[3] Balogh, T., "The holovizio system," in [Stereoscopic Displays and Virtual Reality Systems XIII], 6055, International Society for Optics and Photonics (2006).

[4] Georgiev, T. and Intwala, C., "Light field camera design for integral view photography," Adobe Technical Report (2006).

[5] Magnor, M. and Girod, B., "Data compression for light-field rendering," IEEE Transactions on Circuits and Systems for Video Technology 10(3), 338-343 (2000).

[6] Dufaux, F., Ouaret, M., and Ebrahimi, T., "Recent advances in multiview distributed video coding," in [Mobile Multimedia/Image Processing for Military and Security Applications 2007], 6579, International Society for Optics and Photonics (2007).

[7] Kovács, P. T., Nagy, Z., Barsi, A., Adhikarla, V. K., and Bregovic, R., "Overview of the applicability of H. 264/MVC for real-time light-field applications," in [3DTV-Conference: The True Vision-Capture, Transmission and Display of 3D Video (3DTV-CON), 2014], IEEE (2014).

[8] Balogh, T. and Kovács, P. T., "Real-time 3D light field transmission," in [Real-Time Image and Video Processing 2010], 7724, 772406, International Society for Optics and Photonics (2010).

[9] Cserkaszky, A., Barsi, A., Kara, P. A., and Martini, M. G., "To interpolate or not to interpolate: Subjective assessment of interpolation performance on a light field display," in [Multimedia \& Expo Workshops (ICMEW), 2017 IEEE International Conference on], 55-60, IEEE (2017).

[10] Vagharshakyan, S., Bregovic, R., and Gotchev, A., "Light field reconstruction using shearlet transform," IEEE transactions on pattern analysis and machine intelligence 40(1), 133-147 (2018).

[11] Tanimoto, M., Tehrani, M. P., Fujii, T., and Yendo, T., "Free-viewpoint TV," IEEE Signal Processing Magazine 28(1), 67-76 (2011).

[12] Cserkaszky, A., Barsi, A., Kara, P. A., and Martini, M. G., "Towards display-independent light-field formats," in [3D Immersion (IC3D), 2017 International Conference on], IEEE (2017).

[13] Magalhães, D. S., Serra, R. L., Vannucci, A. L., Moreno, A. B., and Li, L. M., "Glasses-free 3D viewing systems for medical imaging," Optics \&3 Laser Technology 44(3), 650-655 (2012).

[14] Narita, Y., Tsukagoshi, S., Suzuki, M., Miyakita, Y., Ohno, M., Arita, H., Saito, Y., Kokojima, Y., Watanabe, N., Moriyama, N., and Shibui, S., "Usefulness of a glass-free medical three-dimensional autostereoscopic display in neurosurgery," International journal of computer assisted radiology and surgery 9(5), 905-911 (2014). 
[15] Adhikarla, V. K., Sodnik, J., Szolgay, P., and Jakus, G., "Exploring direct 3D interaction for full horizontal parallax light field displays using leap motion controller," Sensors 15(4), 8642-8663 (2015).

[16] Balogh, T. and Kovács, P. T., "Holovizio: The next generation of 3D oil \& gas visualization," in [70th EAGE Conference and Exhibition-Workshops and Fieldtrips], (2008).

[17] Kara, P. A., Martini, M. G., Nagy, Z., and Barsi, A., "Cinema as large as life: Large-scale light field cinema system," in [2017 International Conference on 3D Immersion (IC3D)], IEEE (2017).

[18] Kara, P. A., Cserkaszky, A., Martini, M. G., Barsi, A., Laszlo, B., and Balogh, T., "Evaluation of the Concept of Dynamic Adaptive Streaming of Light Field Video," IEEE Transactions on Broadcasting 64(2), 407-421 (2018). 